\title{
Brittle fracture during folding of rocks: A finite element study
}

\author{
P. Jäger ${ }^{\mathrm{a}}$, S.M. Schmalholz ${ }^{\mathrm{b}}$, D.W. Schmid ${ }^{\mathrm{c}}$ and E. Kuhl ${ }^{\mathrm{d} *}$ \\ ${ }^{a}$ Department of Mechanical Engineering, University of Kaiserslautern, Kaiserslautern, Germany; \\ ${ }^{b}$ Geological Institute, ETH Zürich, Zürich, Switzerland; ${ }^{c}$ Physics of Geological Processes, \\ University of Oslo, Oslo, Norway; ${ }^{d}$ Department of Mechanical Engineering, Stanford University, \\ Stanford, USA
}

(Received 31 December 2007; final version received 26 June 2008)

\begin{abstract}
The goal of the present work is the development of a novel computational analysis tool to elaborate folding-induced fracture of geological structures. Discrete failure of brittle rocks is characterised by three sets of governing equations: the bulk problem, the interface problem and the crack problem. The former two sets which define the deformation field are highly nonlinear and strongly coupled. They are solved iteratively within a Hansbo-type finite element setting. The latter set defines the crack kinematics. It is linear and solved in a single post-processing step. To elaborate the features of the computational algorithm, we define a unique benchmark problem of a single, geometrically nonlinear plate, which is subjected to layer-parallel in-plane compression combined with different levels of superposed in-plane shear. The resulting folding, or buckling, induces brittle failure in the tensile regime. By systematically increasing the shear strain at constant compression, we develop crack deviation angle versus shear-to-compression ratio tables. We determine the corresponding damage zones, analyse the folding modes and elaborate the force versus amplification diagrams. The proposed two-field folding-induced fracture algorithm can ultimately be applied to interpret natural folded rocks and understand their evolution, structural development and histology.
\end{abstract}

Keywords: geophysics; rock mechanics; fracture; folding; finite element method

\section{Fold-fracture relationships in rocks}

Folds are very common structures in nature and occur on many scales, from millimetre to hundreds of kilometres (e.g. [1-4]). The folds discussed in this study are the result of folding, or buckling, which is a mechanical instability that arises during the layer-parallel compression of mechanically strong layers or plates (e.g. [1,5]). Rock units can also be bent without buckling which is sometimes termed forced folding (e.g. [6]); a mechanism not discussed in this study. Especially folded rocks of the upper crust, such as sandstones and limestones, often exhibit fractures and fracture sets with specific orientations relative to the fold geometry, see Figures 1 and 2. The temporal and spatial relationship between folding and fracturing is of great importance for geologists because studying the fold-fracture

\footnotetext{
*Corresponding author. Email: ekuhl@stanford.edu
} 

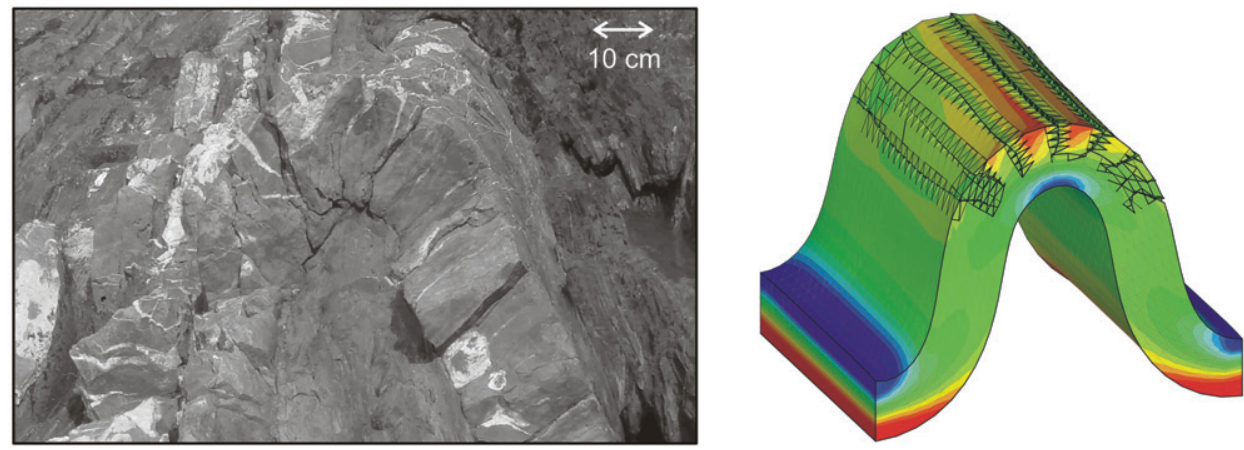

Figure 1. Left: Folded sandstone layer embedded in shale, south-west Portugal. Wedge-shaped dilational fractures filled with quartz are visible at the outer part of the fold hinge. Right: Contour plot of stress during simulation of faulting and fracturing.
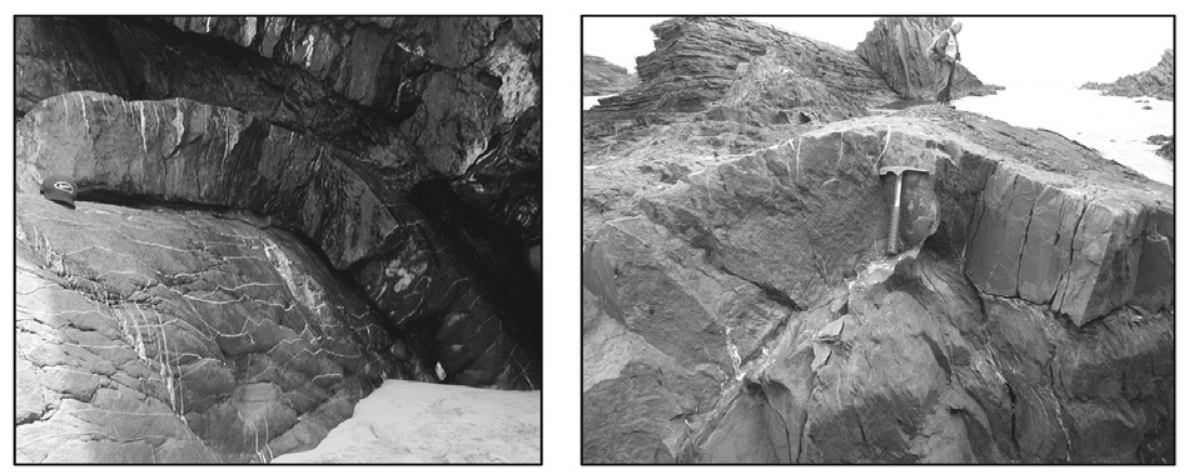

Figure 2. Folded sandstone layers embedded in shale, south-west Portugal. The dilational fracture planes are filled with quartz, and are oriented perpendicular to the bedding and sub-parallel to the fold axes.

relationships improves the understanding of their formation mechanism and allows predicting fracture orientations and fracture densities in natural folded rocks (e.g. [2,6-9]). These predictions are particularly important for industrial applications such as groundwater and hydrocarbon flow because fractures control the permeability of rock units (e.g. $[7,10])$.

Usually, there are several fracture sets with different orientations around folded upper crustal rocks and it is often difficult to determine which fracture sets are related to the folding and which are not (e.g. [2]). Therefore, a main question for field geologists studying fold-fracture relationships is to determine if the fractures in the folded rocks have formed before, during or after folding. Fractures that formed before folding are not related to the characteristic stress field caused by folding but may have introduced some anisotropy in the folded rocks (e.g. [7,10]). Fractures that formed after folding during, for example, the uplift and exhumation of the folded rocks may have orientations completely unrelated to the stress field during folding. However, fractures that formed during folding are expected 
to show orientations that are related to the stress field caused by folding. The fracture orientations that form during folding can be predicted for simple folding scenarios (e.g. $[2,11])$. For example, the two most common dilational fracture sets related to folding are expected to be both perpendicular to the bedding but either parallel or orthogonal to the fold axes (e.g. [2]; see Figure 2). Folding controls not only the orientation of synfolding fractures but also the type of these fractures (e.g. [2,11]). There are two fundamental types of fractures: dilational fractures (also termed extension fractures or joints) and shear fractures (also termed faults, e.g. [12]). Both fracture types can be generated during folding.

In this study, the development of fractures during folding of an elastic plate is numerically simulated in three dimensions. Since no previous numerical studies exist for this problem, a relatively simple model setup is chosen here: (i) the folded plate is free and not embedded in a mechanically weaker medium, (ii) the rheology is purely elastic, (iii) the plate is initially homogeneous and isotropic and no initial fractures exist, and (iv) only dilational fractures are considered using the Rankine criterion.

Although the fold-fracture relationship is important for geologists, there are no numerical models that satisfactorily simulate simultaneous fractures during folding of elastic rocks. One reason presumably is that while folding of an elastic plate is relatively easy to model numerically, fracturing is not. The discontinuous nature of the fracture process typically prohibits the application of classical continuum mechanics concepts and standard finite element based solution techniques. Provided the location of the failure surface is a priori known, interface elements can be introduced locally and fracture can take place at predefined locations between the elements. Arbitrary geological failure zones, however, should not be restricted to inter-element boundaries. They should be able to run right through the elements. Accordingly, discontinuous finite element shape functions have to be introduced. The underlying concept, the celebrated extended finite element method, has only been developed within the last decade (see [13-15]). Within this article, we apply a technique that is somewhat similar to the extended finite element method. As such, it can be understood as a re-parameterization of the extended finite element method in the sense that it uses only displacement degrees of freedom. This technique was initially developed for elastic fracture at small deformations (see $[16,17]$ ) and recently extended to large deformations in a fully three-dimensional setting (see [18-20]). Roughly speaking, we introduce a discontinuity surface and double the degrees of freedom in every element that is identified to fail. The displacement field is then interpolated independently of both sides of this discontinuity.

The method itself is extremely elegant and straightforward to implement. When simulating three-dimensional failure, however, an additional level of complexity arises for both the classical extended finite element method and the method chosen herein: the geometric representation of a smooth three-dimensional failure surface is extremely cumbersome from a computational point of view. Different strategies have been suggested to solve this problem with either local or non-local crack tracking algorithms (e.g. [21,22]). Here, we suggest a fully global crack tracking algorithm based on the introduction of additional global degrees of freedom that ensure the smoothness of the newly generated failure surface (see [18,23]).

The main aims of this study are (i) to present a numerical algorithm that can simulate the simultaneous processes of fracturing and folding in a self-consistent way, (ii) to quantify the temporal evolution of dilational fractures during folding, (iii) to quantify the 
spatial orientation of fractures during folding, (iv) to quantify the impact of layer-parallel shearing, or rather wrenching, on the temporal and spatial formation of fractures, (v) to quantify the impact of fractures on the fold amplification, and (vi) to present a first numerical study for folding controlled fracturing which can be elaborated for more sophisticated practical geological applications in the future. Accordingly, the paper is organised as follows: Section 2 summarises the three sets of governing equations for fracture in folding rocks. In Section 3, we then briefly illustrate the finite element based numerical algorithm embedded in a staggered solution for the potentially discontinuous deformation field and the scalar-valued crack isosurfaces. We then define a unique benchmark problem of a single-layered folding plate and study the six above-mentioned aspects in Section 4. The paper concludes with a final discussion in Section 5.

\section{Continuum mechanics equations for fracture in folding rocks}

The folding algorithm we will describe in this section is essentially governed by three sets of equations: the elastic bulk problem, the cohesive interface problem and the crack tracking problem. The elastic bulk problem and the crack tracking problem are valid in the entire domain $\mathcal{B}$, whereas the cohesive interface problem is valid only on the discontinuity $\Gamma$. Since the extension to multiple discontinuities is straightforward, we restrict the description to one single discontinuity in the sequel. First, we will briefly summarise the corresponding continuous field equations for each of the three problems. Next, we derive their discrete counterpart and illustrate a numerical method to solve them within a finite element framework.

\subsection{The bulk problem}

Let us first characterise the elastic bulk behaviour in the body $\mathcal{B}$, which we assume to be crossed by a discontinuity $\Gamma$. On each side of this discontinuity, i.e. on $\mathcal{B}^{+}$and $\mathcal{B}^{-}$, we introduce an independent set of deformation maps $\varphi$ which maps particles from their original position $\boldsymbol{X}$ in the reference configuration $\mathcal{B}$ to their current position $\boldsymbol{x}$ in the deformed configuration $\mathcal{S}$, as illustrated in Figure 3.

$$
\varphi(X):=\left\{\begin{array}{ll}
\varphi^{+}(X) \\
\varphi^{-}(X)
\end{array} \quad F= \begin{cases}F^{+}=\nabla_{X} \varphi^{+} & \forall X \in \mathcal{B}^{+} \\
F^{-}=\nabla_{X} \varphi^{-} & \forall X \in \mathcal{B}^{-}\end{cases}\right.
$$

Accordingly, we can introduce independent deformation gradients $\boldsymbol{F}^{+}$and $\boldsymbol{F}^{-}$and corresponding Jacobians $J^{+}=\operatorname{det}\left(\boldsymbol{F}^{+}\right)$and $J^{-}=\operatorname{det}\left(\boldsymbol{F}^{-}\right)$on either side of the discontinuity.

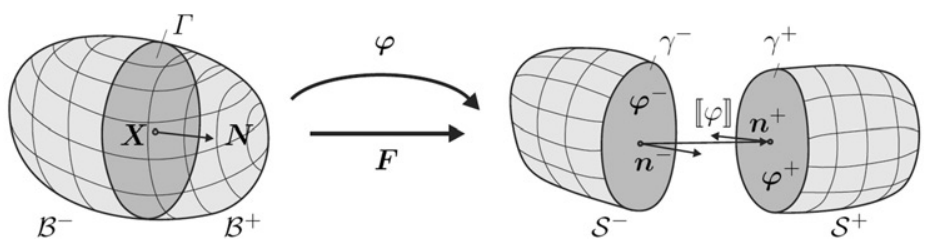

Figure 3. Elastic bulk problem. Independent mappings $\boldsymbol{\varphi}^{+}$and $\boldsymbol{\varphi}^{-}$on both sides $\mathcal{B}^{+}$and $\mathcal{B}^{-}$of the discontinuity $\Gamma$ inherently introduce a jump $[\varphi]$ in the deformation field. 
In the absence of body forces and inertia terms, the equilibrium equation reduces to the vanishing divergence of the Piola stress $\boldsymbol{P}$, which can be stated independently in both subdomains $\mathcal{B}^{+}$and $\mathcal{B}^{-}$:

$$
\operatorname{Div}(\boldsymbol{P})=\mathbf{0} \quad \forall \boldsymbol{X} \in \mathcal{B}^{+} \cup \mathcal{B}^{-} .
$$

On the external boundary $\partial \mathcal{B}$, which can be subdivided into disjoint parts $\partial \mathcal{B}=\partial \mathcal{B}_{u} \cup \partial \mathcal{B}_{t}$ with $\partial \mathcal{B}_{u} \cap \partial \mathcal{B}_{t}=\varnothing$, either Dirichlet boundary conditions $\boldsymbol{\varphi}=\boldsymbol{u}^{\mathrm{P}}$ or Neumann boundary conditions, $\boldsymbol{P} \cdot \boldsymbol{N}=\boldsymbol{T}^{\mathrm{p}}$ can be prescribed in terms of given displacements $\boldsymbol{u}^{\mathrm{p}}$ or given surface tractions $\boldsymbol{T}^{\mathrm{P}}$. We assume a compressible elastic constitutive behaviour of neo-Hookean type inside the bulk. Accordingly, the Cauchy stress $\boldsymbol{\sigma}$ can be expressed in terms of the Lamé parameters $\lambda$ and $\mu$. The Cauchy stress $\boldsymbol{\sigma}$ can be related to the Piola stress $\boldsymbol{P}$ through Nanson's formula. In general, these stresses can take different values on both sides of the discontinuity $\mathcal{B}^{+}$and $\mathcal{B}^{-}$:

$$
\boldsymbol{P}=J \boldsymbol{\sigma} \cdot \boldsymbol{F}^{-\mathrm{t}} \quad \boldsymbol{\sigma}=\frac{1}{J}\left[\lambda \ln (J) \boldsymbol{I}-\mu \boldsymbol{I}+\mu \boldsymbol{F} \cdot \boldsymbol{F}^{\mathrm{t}}\right] \quad \forall \boldsymbol{X} \in \mathcal{B}^{+} \cup \mathcal{B}^{-} .
$$

Following the classical principal stress based Rankine criterion, we solve the eigenvalue problem of the Cauchy stress tensor $\boldsymbol{\sigma}=\sum_{i=1}^{3} \lambda_{i}^{\sigma} \boldsymbol{n}_{i}^{\sigma} \otimes \boldsymbol{n}_{i}^{\sigma}$ and allow for crack propagation if the largest eigenvalue $\lambda_{i}^{\sigma_{\max }}$ exceeds the rupture stress; i.e. $\lambda_{i}^{\sigma_{\max }}>\sigma^{\text {crit }}$. Furthermore, the eigenvector $\boldsymbol{n}_{i}^{\sigma_{\max }}$, related to the maximal eigenvalue, defines the normal to the crack propagation direction $\boldsymbol{n}=\boldsymbol{n}_{i}^{\sigma_{\max }}$ in the spatial configuration. The two remaining eigenvectors $\boldsymbol{t}_{2}=\boldsymbol{n}_{i}^{\sigma_{2}}$ and $\boldsymbol{t}_{3}=\boldsymbol{n}_{i}^{\sigma_{3}}$ related to the second and third eigenvector $\lambda^{\sigma_{\max }}>\lambda^{\sigma_{2}}>\lambda^{\sigma_{3}}$ span the crack plane in the spatial configuration. Their corresponding material unit vectors $\boldsymbol{T}_{2}=\boldsymbol{F}^{-1} \cdot \boldsymbol{t}_{2} /\left|\boldsymbol{F}^{-1} \cdot \boldsymbol{t}_{2}\right|$ and $\boldsymbol{T}_{3}=\boldsymbol{F}^{-1} \cdot \boldsymbol{t}_{3} /\left|\boldsymbol{F}^{-1} \cdot \boldsymbol{t}_{3}\right|$ will prove essential for the kinematic characterisation of the discontinuity surface.

\subsection{The interface problem}

We assume that material failure is exclusively attributed to the cohesive interface, which is characterised through its own independent set of equations. The introduction of two different deformation fields for both sides of the discontinuity $\mathcal{B}^{+}$and $\mathcal{B}^{-}$inherently introduces possible jumps in the deformation map $[[\varphi]]=\varphi^{+}-\varphi^{-}$on the discontinuity, i.e. $\forall \boldsymbol{X} \in \Gamma$.

As illustrated in Figure 4, all points initially located on the unique discontinuity surface $\Gamma$ are mapped onto two surfaces $\gamma^{+}$and $\gamma^{-}$in the deformed configuration. To uniquely characterise discontinuous failure at finite deformations, we apply the concept of

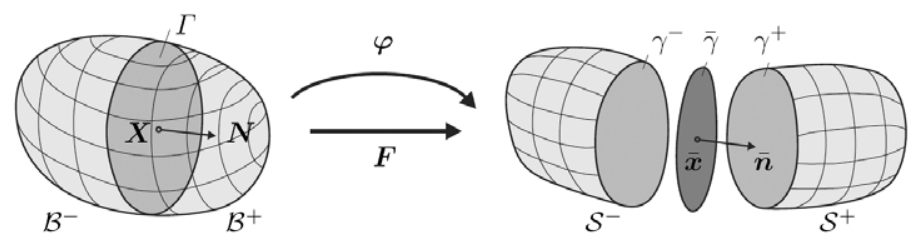

Figure 4. Interface problem. Concept of fictitious discontinuity surface $\bar{\gamma}$ located between the two discontinuity surfaces $\gamma^{+}$and $\gamma^{-}$. 
a fictitious discontinuity $\bar{\varphi}$, which is assumed to be located between the two discontinuity surfaces $\gamma^{+}$and $\gamma^{-}$in the deformed configuration:

$$
\bar{\varphi}:=\frac{1}{2}\left[\boldsymbol{\varphi}^{+}+\boldsymbol{\varphi}^{-}\right] \quad \overline{\boldsymbol{F}}=\frac{1}{2}\left[\boldsymbol{F}^{+}+\boldsymbol{F}^{-}\right] \quad \forall X \in \Gamma .
$$

Again, the corresponding deformation gradient $\overline{\boldsymbol{F}}$ and its Jacobian $\bar{J}=\operatorname{det}(\overline{\boldsymbol{F}})$ follow straightforwardly. The normal vector $\bar{n}$ to the fictitious discontinuity can then be expressed through the classical Nanson formula as $\overline{\boldsymbol{n}}=\bar{J} \overline{\boldsymbol{F}}^{-\mathrm{t}} \cdot \boldsymbol{N}$. On the internal boundary $\Gamma$ (i.e. along the fictitious discontinuity) the equilibrium condition,

$$
\boldsymbol{P}^{+} \cdot \boldsymbol{N}=\boldsymbol{P}^{-} \cdot \boldsymbol{N}=\overline{\boldsymbol{T}} \quad \forall X \in \Gamma,
$$

states that tractions $\overline{\boldsymbol{T}}$ acting on the discontinuity have to be equal in direction and magnitude, however, taking the opposite sign, compare Figure 4. Similar to the Cauchy stresses $\boldsymbol{\sigma}$ in the bulk, the Cauchy tractions $\overline{\boldsymbol{t}}$ on the fictitious discontinuity can be related to the tractions $\overline{\boldsymbol{T}}$ on the undeformed domain through Nanson's formula in terms of the area elements $\mathrm{d} a$ and $\mathrm{d} A$. This presented approach is designated for arbitrary cohesive traction separation laws. For the sake of simplicity, we account for instantaneous brittle failure without any further cohesive resistance. However, to avoid penetration of the crack surfaces during the faulting process, we use the following linear traction separation relation:

$$
\overline{\boldsymbol{T}}=\frac{\mathrm{d} a}{\mathrm{~d} A} \overline{\boldsymbol{t}} \quad \overline{\boldsymbol{t}}=\alpha \frac{E^{\mathrm{int}}}{h^{\mathrm{el}}}\left[\left[\varphi_{n}\right]\right] \overline{\boldsymbol{n}} .
$$

Here, $E^{\text {int }}$ represents the interface stiffness, $h^{\mathrm{el}}$ is the characteristic element size, and $\alpha$ is a dimensionless parameter that takes the interpretation of a penetration penalty. In detail, we set $\alpha=0$ in case of interface tension (i.e. for $\left[\left[\varphi_{n}\right]\right]=[[\varphi]] \cdot \bar{n} \geq 0$ ) when the normal projection of the deformation jump is non-negative. In the case of interface compression (i.e. for $\left[\left[\varphi_{n}\right]\right]=[[\varphi]] \cdot \overline{\boldsymbol{n}}<0$ ) however, we penalise penetration by choosing $\alpha>0$.

\subsection{The crack problem}

The characterisation of smooth three-dimensional failure surfaces is handled through a global crack tracking algorithm, which introduces an additional set of partial differential equations. The general idea is to represent the crack in the form of isosurfaces $\phi=$ const of an additional scalar field $\phi(\boldsymbol{X})$. A particular isosurface of constant value is defined as the kinematic representation of the discrete three-dimensional failure surface, see Oliver et al. [23] and Jäger et al. [18]. Conceptually speaking, the goal of the crack tracking problem is to find the scalar field $\phi(X)$ which is the solution to the following field equation:

$$
\operatorname{Div}(\boldsymbol{j})=0 \quad \forall \boldsymbol{X} \in \mathcal{B},
$$

where the flux vector $\boldsymbol{j}$ is a linear function of the gradient of $\nabla \phi$ :

$$
\boldsymbol{j}=\left[\boldsymbol{T}_{2} \otimes \boldsymbol{T}_{3}+\boldsymbol{T}_{3} \otimes \boldsymbol{T}_{3}\right] \cdot \nabla_{X} \phi \quad \forall \boldsymbol{X} \in \mathcal{B} .
$$

By construction, the particular format for the anisotropic constitutive tensor $\mathbf{D}=$ $\boldsymbol{T}_{2} \otimes \boldsymbol{T}_{2}+\boldsymbol{T}_{3} \otimes \boldsymbol{T}_{3}$ ensures that the flux $\boldsymbol{j}$ is always a weighted linear combination of the tangent vectors $\boldsymbol{T}_{2}$ and $\boldsymbol{T}_{3}$, which, as mentioned before, are the remaining two eigenvectors 
of the Rankine criterion in the reference configuration. Since the anisotropy tensor $\mathbf{D}$ is rank deficient, we apply slight perturbations $\varepsilon$ as $\mathbf{D}=\boldsymbol{T}_{2} \otimes \boldsymbol{T}_{2}+\boldsymbol{T}_{3} \otimes \boldsymbol{T}_{3}+\varepsilon \boldsymbol{I}$ to ensure that the overall system is solvable. The problem of finding isosurfaces $\phi=$ const is obviously a classical boundary value problem in terms of the field $\phi(X)$ characterised through an anisotropic Laplace equation. On the boundary $\partial \mathcal{B}$, which can be subdivided into disjoint parts $\partial \mathcal{B}=\partial \mathcal{B}_{\phi} \cup \partial \mathcal{B}_{j}$ with $\partial \mathcal{B}_{\phi} \cap \partial \mathcal{B}_{j}=\varnothing$, either Dirichlet boundary conditions $\boldsymbol{\phi}=\boldsymbol{\phi}^{\mathrm{p}}$ or Neumann boundary conditions $\boldsymbol{j} \cdot \boldsymbol{N}=j^{\mathrm{p}}$ can be prescribed.

The successful representation of cracks crucially relies on the identification of a particular isosurface $\phi=$ const as discrete failure surface. In general, there are two different strategies to identify a failure surface. For the first approach, an isosurface of a predefined constant level value $\phi=\phi^{\mathrm{p}}$ is defined as failure surface. This description is very well suited for problems with one or two cracks. In this case, homogeneous Dirichlet boundary conditions are applied to the nodes of the crack initialisation elements. Alternatively, for the second approach, the location of crack initialisation is predefined. Corresponding boundary conditions are specified on the geometry. At crack initiation; i.e. when the Rankine criterion is violated, the current value $\phi=\phi^{\text {crit }}$ of the isosurface at the predefined location is used to characterise the failure surface. This approach is advantageous in the case of multiple cracks.

\section{Computational algorithm for fracture in folding rocks}

Next, we illustrate how to solve the governing Equations (2), (5) and (7) within a nonlinear finite element setting. By their very nature, the elastic bulk problem and the cohesive interface problem are strongly coupled. Both, however, are only weakly coupled to the crack tracking problem.

Accordingly, we chose to solve the bulk and interface equilibrium simultaneously and only then solve the crack kinematics in a post-processing step. The corresponding weak forms follow from a multiplication with the test functions $\delta \varphi,[[\delta \varphi]]$ and $\delta \phi$, integration over the domain of interest $\mathcal{B}$ and its discontinuity $\Gamma$, and inclusion of the Neumann boundary conditions $\boldsymbol{P} \cdot \boldsymbol{N}=\boldsymbol{T}^{\mathrm{p}}$ and $\boldsymbol{j} \cdot \boldsymbol{N}=j^{\mathrm{p}}$ :

$$
\begin{aligned}
\int_{\mathcal{B}^{+} \cup \mathcal{B}^{-}} \delta \boldsymbol{F}: \boldsymbol{P} \mathrm{d} V+\int_{\Gamma}[[\delta \boldsymbol{\varphi}]] \cdot \overline{\boldsymbol{T}} \mathrm{d} A & =\int_{\partial \mathcal{B}_{t}} \delta \boldsymbol{\varphi} \cdot \boldsymbol{T}^{\mathrm{p}} \mathrm{d} A \\
\int_{\mathcal{B}} \nabla_{\boldsymbol{X}} \delta \phi \cdot \boldsymbol{j} \mathrm{d} V & =\int_{\partial \mathcal{B}_{j}} \delta \phi j^{\mathrm{p}} \mathrm{d} A .
\end{aligned}
$$

For the finite element formulation, it proves convenient to distinguish between standard continuous elements and discontinuous elements which are crossed by the discontinuity surface. For the continuous elements, we apply a standard interpolation of the test functions $\delta \boldsymbol{\varphi}$, the deformation field $\boldsymbol{\varphi}$, and their gradients $\delta \boldsymbol{F}$ and $\boldsymbol{F}$. For the discontinuous elements, we apply an independent interpolation of the deformation field $\varphi^{+}$and $\varphi^{-}$and its gradient $\boldsymbol{F}^{+}$and $\boldsymbol{F}^{-}$on the individual sides of the discontinuity $\mathcal{B}^{+}$and $\mathcal{B}^{-}$. Conceptually speaking, both deformation fields $\varphi^{+}$and $\varphi^{-}$are interpolated independently over the element. To this end, we essentially double the degrees of freedom of the entire element. The interpolated fields are then set to zero on one side of the discontinuity, while they take their usual values on the other side. For the crack tracking problem, 
we apply a standard linear interpolation of the test and trial functions $\delta \phi$ and $\phi$ and their gradients $\nabla \delta \phi$ and $\nabla \phi$ :

$$
\begin{aligned}
& \delta \boldsymbol{\varphi}=\sum_{i} \delta \boldsymbol{\varphi}_{i} N^{i} \quad \delta \boldsymbol{F}=\sum_{i} \delta \boldsymbol{\varphi}_{i} \otimes \nabla_{\boldsymbol{X}} N^{i} \quad \boldsymbol{\varphi}=\sum_{j} \boldsymbol{\varphi}_{j} N^{j} \quad \boldsymbol{F}=\sum_{j} \boldsymbol{\varphi}_{j} \otimes \nabla_{\boldsymbol{X}} N^{j} \\
& {[[\delta \varphi]]=\sum_{\bar{i}} \delta \boldsymbol{\varphi}_{\bar{i}} \bar{N}^{\bar{i}} \quad \delta \overline{\boldsymbol{F}}=\sum_{\bar{i}} \delta \boldsymbol{\varphi}_{\bar{i}} \otimes \nabla_{\boldsymbol{X}} \bar{N}^{\bar{i}} \quad[[\boldsymbol{\varphi}]]=\sum_{\bar{j}} \boldsymbol{\varphi}_{\bar{j}} \bar{N}^{\bar{j}} \quad \overline{\boldsymbol{F}}=\sum_{\bar{j}} \boldsymbol{\varphi}_{\bar{j}} \otimes \nabla_{\boldsymbol{X}} \bar{N}^{\bar{j}}} \\
& \delta \phi=\sum_{i} \delta \phi_{i} N^{i} \quad \nabla_{\boldsymbol{X}} \delta \phi=\sum_{i} \delta \phi_{i} \otimes \nabla_{\boldsymbol{X}} N^{i} \quad \phi=\sum_{j} \phi_{j} N^{j} \quad \nabla_{\boldsymbol{X}} \phi=\sum_{j} \phi_{j} \otimes \nabla_{\boldsymbol{X}} N^{j} .
\end{aligned}
$$

Here, $N^{i}$ and $N^{j}$ are the standard shape functions for tetrahedral elements and $i$ and $j$ are the four tetrahedral nodes. To unify the notation, we have introduced the sets $\bar{N}^{\bar{i}}$ and $\bar{N}^{\bar{j}}$ which consist of the element shape functions $N$ evaluated on $\Gamma$ multiplied by the corresponding algebraic sign. The overbars indicate the discontinuous elements, for which we have doubled the degrees of freedom such that $\bar{i}$ and $\bar{j}$ are the doubled four, i.e. eight, tetrahedral nodes. Accordingly, $\nabla_{X} \bar{N}$ denotes the gradient of the shape functions $N$ evaluated on the discontinuity $\Gamma$, weighted by the factor $1 / 2$. With the help of the above introduced discretisations, the weak forms (9) can be cast into the following discrete residual statements:

$$
\begin{aligned}
& \mathbf{R}_{I}^{\varphi}=\stackrel{n_{e=1}}{\mathbf{n}} \int_{\mathcal{B}_{e} \cup \mathcal{B}_{d}^{+,-}} \nabla_{\mathrm{X}} N^{i} \cdot \boldsymbol{P} \mathrm{d} V-\int_{\partial \mathcal{B}_{t e}} N^{i} \boldsymbol{T}^{\mathrm{p}} \mathrm{d} A+\int_{\bar{\Gamma}} \bar{N}^{i} \overline{\boldsymbol{T}}([[\boldsymbol{\varphi}]]) \mathrm{d} \bar{A} \doteq \mathbf{0} \\
& \mathrm{R}_{I}^{\phi}=\stackrel{n_{e=1}}{\mathbf{n}} \int_{\mathcal{B}_{e}} \nabla_{\mathrm{X}} N^{i} \cdot \boldsymbol{j} \mathrm{d} V-\int_{\partial B_{j e}} N^{i} j^{\mathrm{p}} \mathrm{d} A \doteq \mathbf{0} .
\end{aligned}
$$

Herein, the operator $\mathbf{A}_{e=1}^{n_{e l}}$ denotes the assembly of all element contributions including the continuous and the discontinuous ones. The first residual $\mathbf{R}_{I}^{\varphi}$ is nonlinear in the unknown deformation field $\varphi(X)$, whereas the second residual $\mathrm{R}_{I}^{\phi}$ is linear in the unknown field $\phi(X)$. The nonlinear set of Equation $(11)_{1}$ is solved numerically based on an incremental iterative Newton-Raphson scheme with the incremental stiffness matrix $\mathbf{K}_{I J}^{\varphi}=\partial \mathbf{R}_{I}^{\varphi} / \partial \boldsymbol{\varphi}_{J}$. The system matrix of the linear set of Equation $(11)_{2}$ follows accordingly, as $\mathrm{K}_{I J}^{\phi}=\partial \mathrm{R}_{I}^{\phi} / \partial \phi_{J}$ :

$$
\begin{aligned}
\mathbf{K}_{I J}^{\varphi} & =\underset{e=1}{n_{e l}} \int_{\mathcal{B}_{e} \cup \mathcal{B}_{d}^{+,-}} \nabla_{\mathrm{X}} N^{i} \cdot\left[\partial_{\boldsymbol{F}} \boldsymbol{P}\right] \cdot \nabla_{\boldsymbol{X}} N^{j} \mathrm{~d} V+\int_{\bar{\Gamma}} \bar{N}^{i}\left[\partial_{\overline{\boldsymbol{F}}} \overline{\boldsymbol{T}}\right] \cdot \nabla_{\boldsymbol{X}} \bar{N}^{j}+\bar{N}^{i}\left[\partial_{[[\varphi]]} \overline{\boldsymbol{T}}\right] \overline{N^{j}} \mathrm{~d} \bar{A} \\
\mathrm{~K}_{I J}^{\phi} & =\underset{e=1}{n_{e l}} \int_{\mathcal{B}_{e}} \nabla_{\mathrm{X}} N^{i} \cdot\left[\partial_{\nabla \phi} \boldsymbol{j}\right] \cdot \nabla_{\boldsymbol{X}} N^{j} \mathrm{~d} V .
\end{aligned}
$$

The solution renders the incremental update of the deformation field $\mathrm{d} \boldsymbol{\varphi}_{J}=\sum_{I=1}^{n_{n p}} \mathbf{K}_{I J}^{\varphi-1} \mathbf{R}_{I}^{\varphi}$ and the crack tracking field $\phi_{J}=\sum_{I=1}^{n_{n p}} \mathrm{~K}_{I J}^{\phi-1} \mathrm{R}_{I}^{\phi}$ for all $n_{n p}$ nodes. Recall that due to the chosen discretisation scheme, the number of global node points $n_{n p}$, which consists of the standard nodes and the duplicated node points for the discontinuous elements increases progressively during ongoing crack propagation. The terms in brackets, i.e. the fourth, third and second order tensors $\left[\partial_{\boldsymbol{F}} \boldsymbol{P}\right],\left[\partial_{\overline{\boldsymbol{F}}} \overline{\boldsymbol{T}}\right],\left[\partial_{[[\varphi]]} \overline{\boldsymbol{T}}\right]$ and $\left[\partial_{\nabla \phi} \boldsymbol{J}\right]$ depend on the choice 

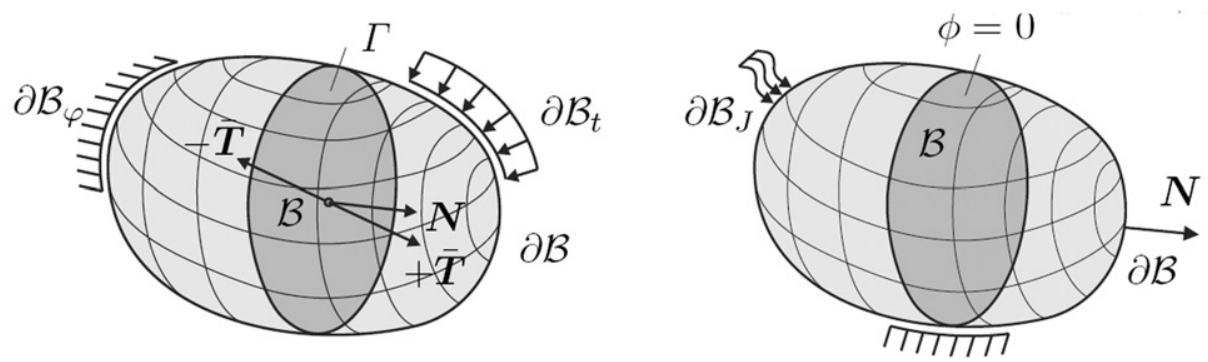

Figure 5. Left: Boundary value problem for bulk $\mathcal{B}$ and interface $\Gamma$. On the external boundary $\partial \mathcal{B}$, either Dirichlet boundary conditions $\varphi=\varphi^{\mathrm{p}}$ or Neumann boundary conditions $\boldsymbol{P} \cdot \boldsymbol{N}=\boldsymbol{T}^{\mathrm{p}}$ can be prescribed. Right: Boundary value problem for crack tracking within the body $\mathcal{B}$. On the external boundary $\partial \mathcal{B}$, either Dirichlet boundary conditions $\phi=\phi^{\mathrm{p}}$ or Neumann boundary conditions $\boldsymbol{j} \cdot \boldsymbol{N}=j^{\mathrm{p}}$ can be prescribed.

of the constitutive equations for the stresses $\boldsymbol{P}$ in the continuous body (3), for the tractions $\boldsymbol{T}$ on the discontinuity surface (6) and for the flux $\boldsymbol{j}$ in the domain (8). For the particular choices suggested in (3) and (6), these linearisations are given, e.g. by Jäger et al. [18]. Since the flux is constant in Equation (8) with $\left[\partial_{\nabla \phi} \boldsymbol{j}\right]=\mathbf{D}$, the crack tracking problem is linear in $\phi(X)$ and can thus be solved without further iteration once the nonlinear deformation problem is solved. Note that the linear interpolation of $\phi(\boldsymbol{X})$ implies that the discrete isosurface takes an element-wise planar representation.

\section{Simulation of fracture in folding rocks}

In this final section, we elaborate the features of the proposed failure algorithm in the context of brittle fracture during folding of rocks. To this end, we carry out a systematic series of numerical experiments (i) to validate that the suggested algorithm can simulate the simultaneous processes of fracturing and folding in a self-consistent way, (ii) to quantify the temporal evolution of dilational fractures during folding, (iii) to quantify the spatial orientation of fractures during folding, (iv) to quantify the impact of layer-parallel shearing or rather wrenching on the temporal and spatial formation of fractures, (v) to quantify the impact of fractures on the fold amplification, and (vi) to present a first numerical study for folding controlled fracturing which can be elaborated for more sophisticated practical geological applications in the future.

\subsection{Model problem for simultaneous fracturing and folding}

All numerical simulations in this paper are based on the unified benchmark problem of a single plate with dimensions $L=10 \mathrm{~m}, B=5 \mathrm{~m}$ and $H=1 \mathrm{~m}$. To trigger a geometric instability, we initialize the plate with a sinusoidal deformation $u_{z}^{\text {init }}=u^{\text {init }} \sin (\pi X / L)$ where $u^{\text {init }}=0.1 \mathrm{~m}$ as illustrated in Figure 6 .

For the spatial discretisation, we choose linear tetrahedral elements. Linear tetrahedra allow an efficient implementation, especially in the context of the numerical integration over the fictitious discontinuity surface. Due to the use of linear interpolations, the discrete discontinuity surface takes an elementwise planar patchwise representation. We discretise the folding plate with 39,360 elements introducing a total of 23,814 degrees of freedom. 
In geodynamic modelling, the folding plate is typically embedded in a weaker medium. For simplicity, however, we assume that the plate is free to move in the thickness direction. This is a valid end-member case if the folding rock layer is mechanically significantly stronger than the embedding rocks. On the lower left and right edge, however, the plate is supported vertically. We thus apply homogeneous Dirichlet boundary conditions $u_{z}=0$ in thickness direction as indicated in Figure 6. Plate folding is initiated through displacement driven in-plane compression and superposed shear on the entire left and right side of the plate. To elaborate the impact of layer-parallel shearing on the temporal and spatial formation of fractures, we define seven individual load cases. Each load case is characterised through the same amount of in-plane compression $u_{x}= \pm 0.01 \mathrm{~m}$ while the in-plane shear $u_{y}$ is varied systematically. Table 1 summarises load cases I-VII classified in terms of the shear-to-compression ratio $u_{y}: u_{x}$. Since we allow multiple cracking, we choose the second strategy of failure surface initialisation as discussed in Section 2.3. Accordingly, failure surfaces are introduced at predefined locations as soon as the Rankine criterion is violated locally. We introduce potential crack root elements on the top of the plate $(Z=H)$ in the middle element $(Y=L / 4)$ of every other row $(X=$ const). By doing so, we allow multiple crack propagation on the one hand and ensure continuity of the potential crack paths on the other hand. For the sake of completeness, we choose to apply Dirichlet boundary conditions of $\phi= \pm 100$ at the upper left and upper right edge to be able to solve the crack tracking problem.

Initially, the plate rheology is purely elastic, it is homogeneous and no initial fracture exists. For the elastic material parameters of the plate, we choose $\lambda=2 \cdot 10^{10} \mathrm{~Pa}$ and $\mu=2 \cdot 10^{10} \mathrm{~Pa}$, corresponding to Young's modulus $E=5 \cdot 10^{10} \mathrm{~Pa}$ and Poisson's ratio to $v=0.25$ which are suitable parameters to describe sandstone or limestone, see e.g. [4]. For the rupture stress we assume a value of $\sigma^{\text {crit }}=0.25 \cdot 10^{10} \mathrm{~Pa}$. The interface stiffness is chosen to $E^{\mathrm{int}}=E=5 \cdot 10^{10} \mathrm{~Pa}$. For our particular finite element discretisation, the

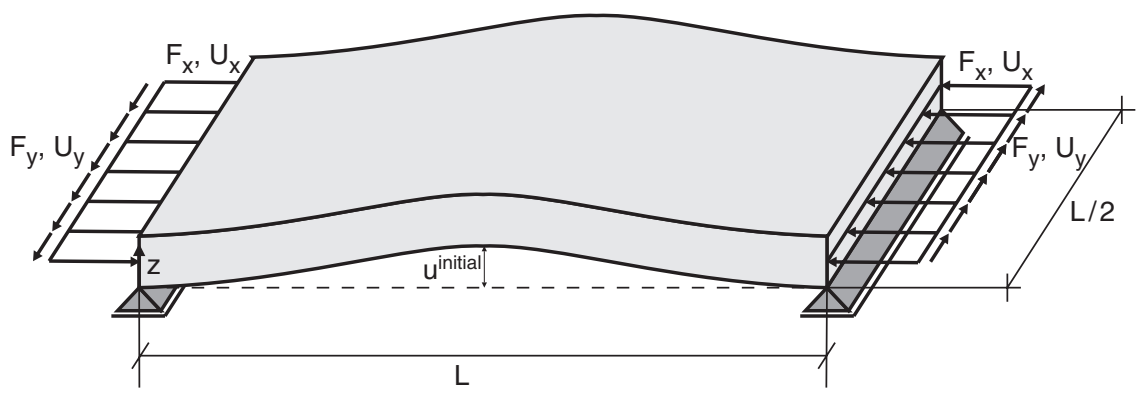

Figure 6. Benchmark problem of single-layered folding plate: Geometry and spatial dimensions.

Table 1. Definition of load cases I-VII in terms of the shear-to-compression ratio $u_{y}: u_{x}$.

\begin{tabular}{lcccccccc}
\hline Load case & & I & II & III & IV & V & VI & VII \\
\hline In-plane compression & $u_{x}$ & 0.01 & 0.01 & 0.01 & 0.01 & 0.01 & 0.01 & 0.01 \\
In-plane shear & $u_{y}$ & 0 & 0.0025 & 0.005 & 0.01 & 0.02 & 0.03 & 0.04 \\
Shear-to-compression ratio & $u_{y}: u_{x}$ & 0 & $1 / 4$ & $1 / 2$ & 1 & 2 & 3 & 4 \\
\hline
\end{tabular}


characteristic element length is $h^{\mathrm{el}}=0.1 \mathrm{~m}$ and the penalty parameter $\alpha$ has been chosen to $\alpha=0.005$, such that $\alpha / h^{\mathrm{el}}=0.05(1 / \mathrm{m})$. This particular choice of the penalty parameter $\alpha$ had been found reasonable during various different case studies.

\subsection{Temporal evolution of dilational fractures during folding}

To elaborate aim (ii), we observe the temporal sequence of emerging cracks during a computation cycle. We identify a total of seven emerging cracks for load cases I-V, and nine cracks for load cases VI and VII. The chronological order of emerging cracks is illustrated in Figure 7. The analysed crack initiation sequence corresponds to an imposed maximum in-plane compression of $u_{x}= \pm 0.7 \mathrm{~m}$ on each side. Surprisingly, the temporal order of emerging cracks is identical in all seven load cases. Table 2 summarises the amount of compression $u_{x}$ at crack initiation for each of the seven cracks based on the numbers introduced in Figure 7. Obviously, the compression deformation intervals $u_{x}$ between the emerging cracks decrease with increasing shear deformation $u_{y}$. As the shearto-compression ratio increases, we observe a smooth transition from plain tensile failure in load case I to a shear dominated failure in load case VII. For the plain compression case I,

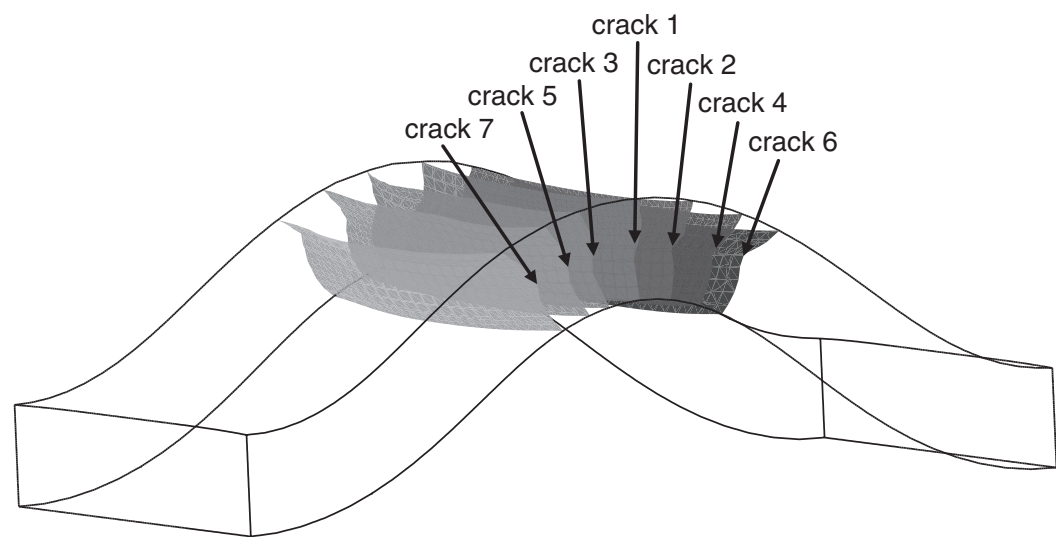

Figure 7. Chronological order of the emerging cracks displayed for their potential crack surfaces.

Table 2. Imposed compression $u_{x}[\mathrm{~m}]$ at crack initiation for all seven cracks $1-7$ and all load cases I-VII.

\begin{tabular}{lccccccc}
\hline Load case & I & II & III & IV & V & VI & VII \\
\hline Shear-to-compression ratio & 0 & $1 / 4$ & $1 / 2$ & 1 & 2 & 3 & 4 \\
$u_{x} @$ crack 1 & 0.28 & 0.28 & 0.28 & 0.27 & 0.25 & 0.23 & 0.22 \\
$u_{x} @$ crack 2 & 0.29 & 0.29 & 0.29 & 0.28 & 0.26 & 0.24 & 0.22 \\
$u_{x} @$ crack 3 & 0.30 & 0.30 & 0.29 & 0.29 & 0.27 & 0.24 & 0.22 \\
$u_{x} @$ crack 4 & 0.33 & 0.33 & 0.33 & 0.33 & 0.29 & 0.26 & 0.22 \\
$u_{x} @$ crack 5 & 0.35 & 0.35 & 0.34 & 0.33 & 0.30 & 0.26 & 0.23 \\
$u_{x} @$ crack 6 & 0.53 & 0.53 & 0.54 & 0.49 & 0.38 & 0.29 & 0.26 \\
$u_{x} @$ crack 7 & 0.59 & 0.58 & 0.57 & 0.51 & 0.38 & 0.29 & 0.26 \\
\hline
\end{tabular}


Table 3. Crack origin positions $X^{\text {orig }}[\mathrm{m}]$ at crack initialisation for all seven cracks $1-7$ and all load cases I-VII.

\begin{tabular}{|c|c|c|c|c|c|c|c|}
\hline Load case & I & II & III & IV & V & VI & VII \\
\hline ar-to-compres & 0 & $1 / 4$ & $1 / 2$ & 1 & 2 & 3 & 4 \\
\hline$X^{\text {orig }} @$ crack 1 & 5.00 & 5.00 & 4.99 & 5.00 & 5.00 & 4.99 & 5.01 \\
\hline$X^{\text {orig }} @$ crack 2 & 5.52 & 5.51 & 5.51 & 5.51 & 5.50 & 5.48 & 5.49 \\
\hline$X^{\text {orig }} @$ crack 3 & 4.49 & 4.49 & 4.49 & 4.49 & 4.49 & 4.50 & 4.53 \\
\hline$X^{\text {orig }} @$ crack 4 & 6.04 & 6.04 & 6.03 & 6.03 & 6.00 & 6.00 & 5.98 \\
\hline$X^{\text {orig }} @$ crack 5 & 3.96 & 3.96 & 3.97 & 3.98 & 3.99 & 4.00 & 4.06 \\
\hline$X^{\text {orig }} @$ crack 6 & 6.58 & 6.58 & 6.58 & 6.56 & 6.51 & 6.49 & 6.45 \\
\hline$X^{\text {orig }} @$ crack 7 & 3.41 & 3.41 & 3.41 & 3.42 & 3.47 & 3.51 & 3.58 \\
\hline$X_{\max }^{\text {orig }}-X_{\min }^{\text {orig }}$ & 3.17 & 3.17 & 3.17 & 3.14 & 3.04 & 2.98 & 2.88 \\
\hline
\end{tabular}

we observe the first crack initiation in the tensile zone right in the middle of the upper plate surface, see crack number 1 in Figure 7. After this crack initiation, the stress state is partially relaxed and the maximum tensile stress gradually moves from the centre to the left and to the right. Next, a set of cracks, numbered 2 and 3 in Figure 7, is initiated left and right to the first crack. Theoretically, these two cracks should emerge simultaneously because of the symmetric geometry of the problem setup. Due to our particular structured triangular discretisation, however, the mesh is biased such that crack 2 on the right is initiated slightly before crack 3 on the left. Crack sets 4 and 5 and then sets 6 and 7 evolve in a similar way. If now, shear is applied in addition to compression, the load increment between the onset of cracking decreases remarkably. In the extreme case VII with a shear-to-compression ratio of $u_{y}: u_{x}=4$, all seven cracks emerge almost simultaneously. This example nicely illustrates the remarkable influence of the shear-to-compression ratio, or rather of the stress state in the plate, on the dominant failure mechanism and thus on the chronological evolution of failure surfaces.

In addition to the temporal sequence of emerging cracks, it is interesting to analyse the spatial origin $x^{\text {orig }}$ of the emerging cracks. Recall that we prescribe the potential location of the crack root, however, we do not know where exactly the crack will emerge. The final location of all crack origins is a quantitative measure for the plate failure in the form of damage. Table 3 displays the widths of the damaged zone $X_{\max }^{\text {orig }}-X_{\min }^{\text {orig }}$ for all seven load cases, which, for our particular problem, is equal to the distance between the origin of crack 6 and 7. Here, we have defined the $X$-width of the projection of the cracked area onto the original $X, Y$-plane in the reference configuration as a qualitative measure for the damaged area. For load case $\mathrm{I}$ it takes a maximum width of $3.17 \mathrm{~m}$ decreasing to a damage zone width of $2.88 \mathrm{~m}$ for load case VII.

\subsection{Spatial orientation of fractures during folding}

Next we address point (iii); i.e. the spatial orientation of the failure surfaces during folding. At first we can use the potential crack isosurfaces visible at the outer boundary as illustrated in Figure 8 for load case I. The cracks clearly trace the direction perpendicular 

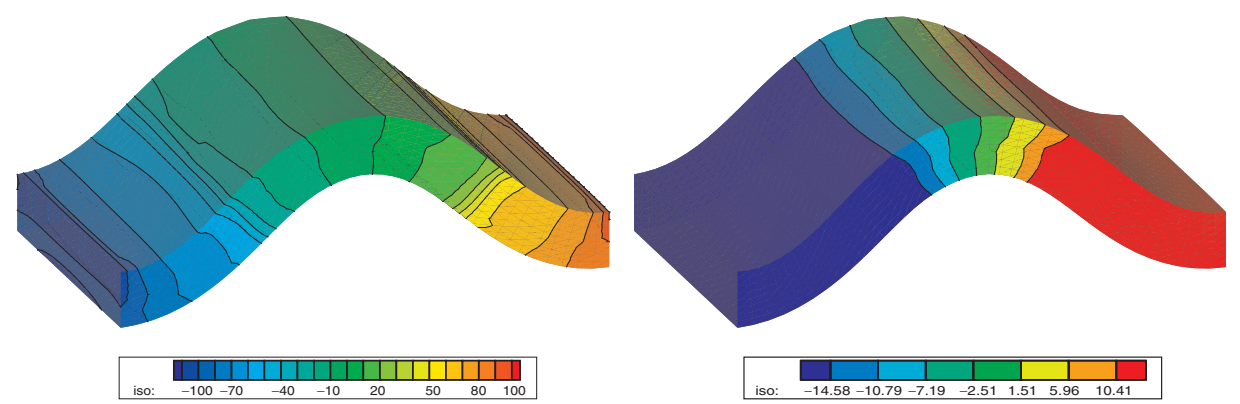

Figure 8. Crack tracking problem: Isosurfaces representing potential crack directions. Left: Load case I for entire range of $\phi$ values with Dirichlet boundary conditions $\phi= \pm 100$ on left and right boundary. Right: Representation of root $\phi$ isosurfaces corresponding to cracks 1-7.

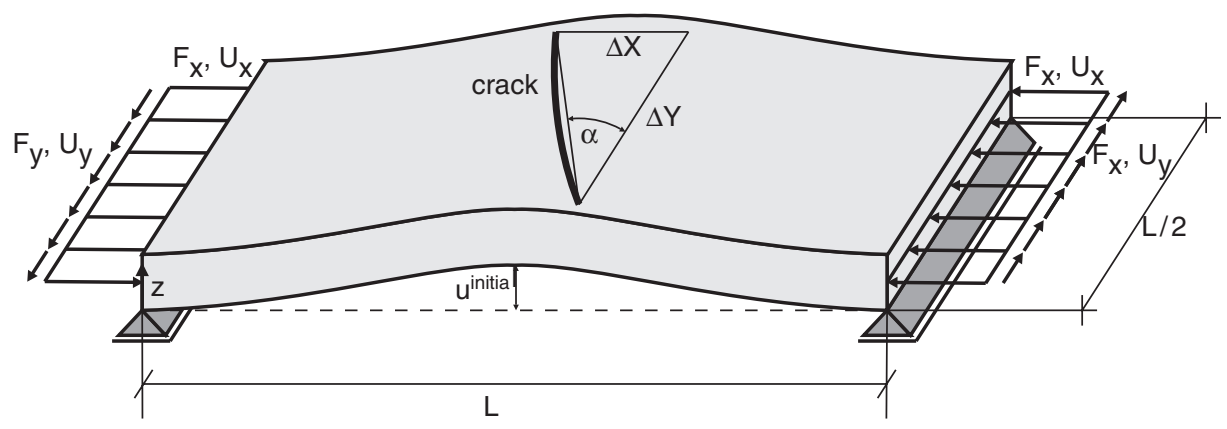

Figure 9. Measurement of the crack deviation angle $\alpha$.

to the principal Cauchy stress as determined through the Rankine criterion. For the displayed plain compression case, discrete failure surfaces are introduced element wise in the direction orthogonal to the maximum principle stress direction; i.e. perpendicular to the compression axis.

\subsection{Impact of layer-parallel shearing on temporal and spatial formation of fractures}

In addition to the qualitative isosurfaces of the previous subsection, we quantify fracture in terms of the crack deviation angle for the emerging cracks. This angle is measured in reference to our fixed coordinate system and thereby address question (iv); i.e. the impact of layer-parallel shearing on the temporal and spatial formation of fracture. In detail we use the two endpoints of each emerging crack to compute its actual length and its orientation with respect to the fixed $Y$-axis, see Figure 9.

The measured angles are summarised in Table 4. For load case I (i.e. the case of pure compression) the crack deviation angle with respect to the $Y$-axis is zero. The principal stress is parallel to the $X$-axis and the crack is oriented perpendicular to the loading axis. As the shear-to-compression ratio is increased from $u_{y}: u_{x}=0$ to 4 , the average crack deviation angle $\alpha$ increases gradually. To clarify the influence of imposed shear 
Table 4. Crack deviation angle $\alpha\left[^{\circ}\right]$ for for all seven cracks $1-7$ and all load cases I-VII.

\begin{tabular}{lccccccc}
\hline Load case & I & II & III & VI & V & VI & VII \\
\hline Shear-to-compression ratio & 0 & $1 / 4$ & $1 / 2$ & 1 & 2 & 3 & 4 \\
$\alpha @$ crack 1 & 0.0 & 1.6 & 3.4 & 5.2 & 8.5 & 11.6 & 51.7 \\
$\alpha @$ crack 2 & 0.0 & 1.7 & 3.2 & 4.0 & 8.0 & 11.5 & 51.4 \\
$\alpha @$ crack 3 & 0.0 & 0.4 & 2.2 & 4.1 & 8.0 & 11.4 & 50.8 \\
$\alpha @$ crack 4 & 0.0 & 1.6 & 2.4 & 5.2 & 8.8 & 16.5 & 63.9 \\
$\alpha @$ crack 5 & 0.0 & 1.5 & 2.1 & 4.1 & 8.9 & 13.8 & 50.4 \\
$\alpha @$ crack 6 & 0.0 & 2.7 & 4.5 & 8.8 & 16.3 & 26.1 & 54.2 \\
$\alpha @$ crack 7 & 0.0 & 3.6 & 5.5 & 8.6 & 12.4 & 20.8 & 49.6 \\
Average $\alpha$ & 0.0 & 1.9 & 3.3 & 5.7 & 10.1 & 15.9 & 53.1 \\
\hline
\end{tabular}

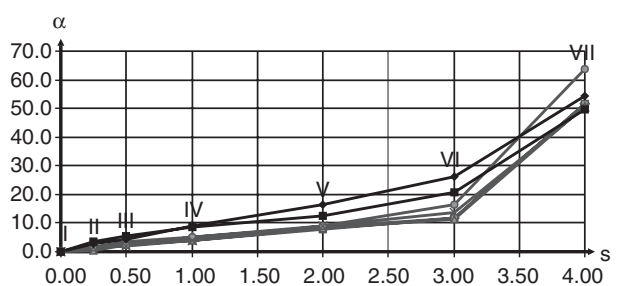

$$
\begin{gathered}
\longrightarrow \text { crack } 1 \rightarrow \text { crack } 2 \multimap \operatorname{crack} 3 \multimap \operatorname{crack} 4 \\
* \text { crack } 5 \rightarrow \operatorname{crack} 6 \rightarrow \text { crack } 7
\end{gathered}
$$

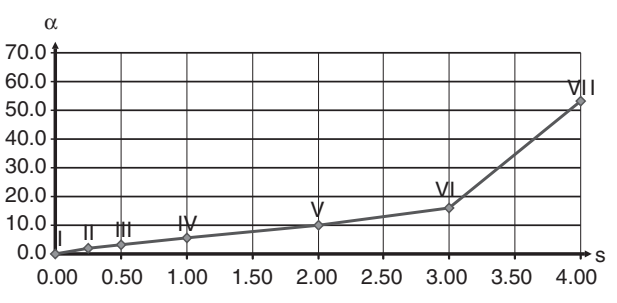

$\rightarrow$ average crack

Figure 10. Crack deviation angle $\left.\alpha{ }^{\circ}\right]$ vs. shear-to-compression ratio. Left: For all seven cracks 1-7 for all load cases I-VII. Right: Average crack deviation angle for all load cases I-VII.

displacement on the spatial formation of fracture, we display this relation for each of the cracks I-VII and for the average crack deviation angle $\alpha$ in Figure 10.

Remarkably, the average crack deviation angle increases linearly from $\alpha=0.0^{\circ}$ for load case I of plain compression to $\alpha=15.9^{\circ}$ for load case VI at a shear-to-compression ratio of $u_{y}: u_{x}=3$. For higher shear-to-compression ratios, however, the crack deviation angle increases nonlinearly. At a shear-to-compression ratio of $u_{y}: u_{x}=4$, the average crack deviation angle is measured to $\alpha=53.1^{\circ}$. At crack initiation, tensile stresses dominate the failure mode for the first six load cases. For load case I, failure occurs under pure tension while for load cases II-VI, shear stresses are superposed in addition. For load case VII, however, shear stresses clearly dominate the failure mechanism. It is interesting that the relation between the crack deviation angle and the shear-to-compression ratio is linear, as long as the dominant deformation mode during crack formation is folding due to the compression.

\subsection{Impact of fractures on fold amplification}

According to our list of aims we finally address item (v) and quantify the impact of fractures on fold amplification. To illustrate the evolution and development of folding, Figure 11 displays a typical time sequence of folding for load case I of pure compression. 


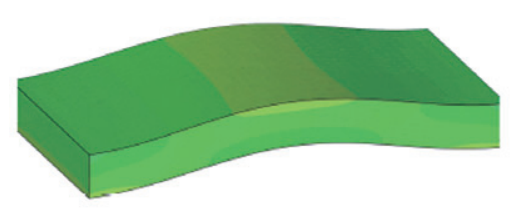

$\mathrm{u}_{\mathrm{x}}=0.2 \mathrm{~m}$

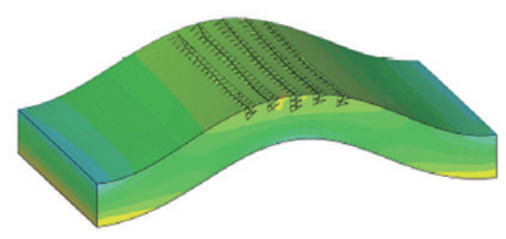

$\mathrm{u}_{\mathrm{x}}=0.4 \mathrm{~m}$

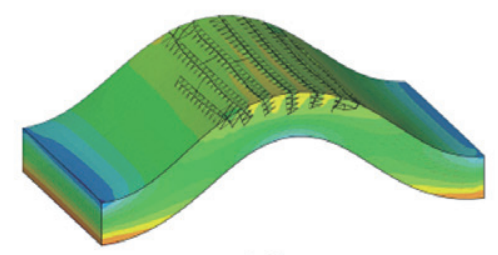

$u_{x}=0.6 m$

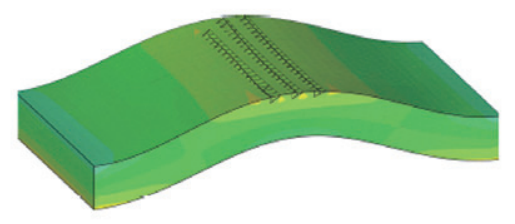

$\mathrm{u}_{\mathrm{x}}=0.3 \mathrm{~m}$

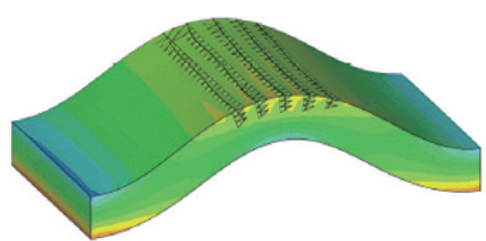

$\mathrm{u}_{\mathrm{x}}=0.5 \mathrm{~m}$

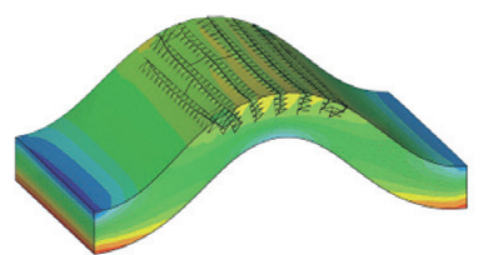

$\mathrm{u}_{\mathrm{x}}=0.7 \mathrm{~m}$

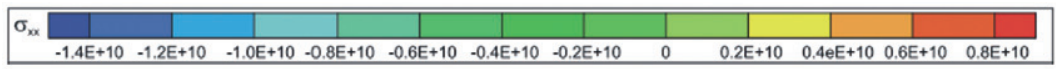

Figure 11. Normal component of Cauchy stress $\sigma_{x x}$ in direction of compression displayed for load case I at different load stages $a-f$.

For this load scenario, all cracks form perpendicular to the loading direction. Clearly, the Cauchy stress and the amplification (i.e. the maximum out-of-plane deformation) increase upon increased compression.

In addition, Figure 12 shows a snapshot for load cases II-VII for the stage at which all seven cracks have developed, compare Table 2. On the one hand Figure 12 nicely illustrates the crack deviation angles for the different load cases. On the other hand it clearly displays that the amplification decreases with increasing shear-to-compression ratio. The depicted folding patterns provide a clear explanation of the decrease in amplification. For plain compression in load case I, the plate buckles in one direction and one single fold forms as triggered by the initial sinusoidal deformation. For increased shear-to-compression ratios, however, the fold tends to rotate away from the compression axis. For the final load cases VII at a shear-to-compression ratio of $u_{y}: u_{x}=4$, the formation of two smaller folds can be observed. These, of course, are smaller in amplification than the single fold in the previous load cases. The multifold buckling of load case VII is an extremely complex failure phenomenon the simulation of which is computationally challenging. For this load case, maximum tensile stresses are not only restricted to the top of the plate and potential crack root elements should also be introduced on the plate bottom. It is thus not surprising that for load case VII, the algorithm fails to capture a further load increase. 


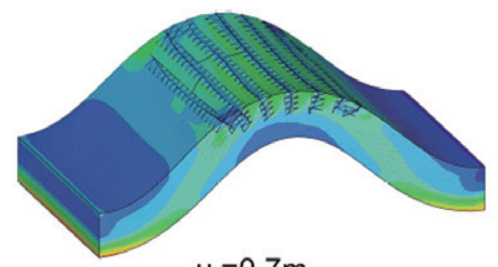

$\mathrm{u}_{\mathrm{x}}=0.7 \mathrm{~m}$
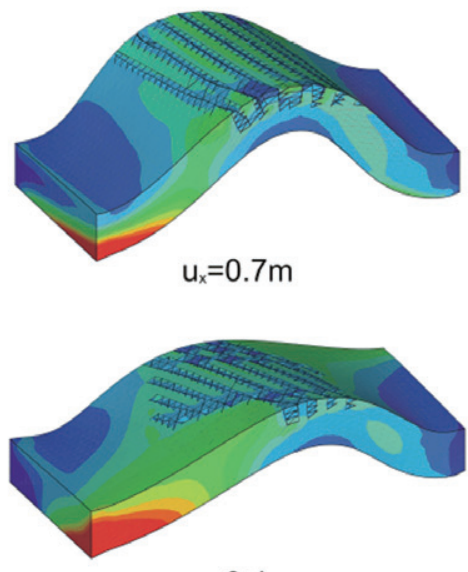

$\mathrm{u}_{\mathrm{x}}=0.4 \mathrm{~m}$

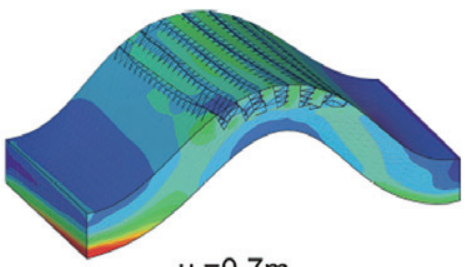

$\mathrm{u}_{\mathrm{x}}=0.7 \mathrm{~m}$
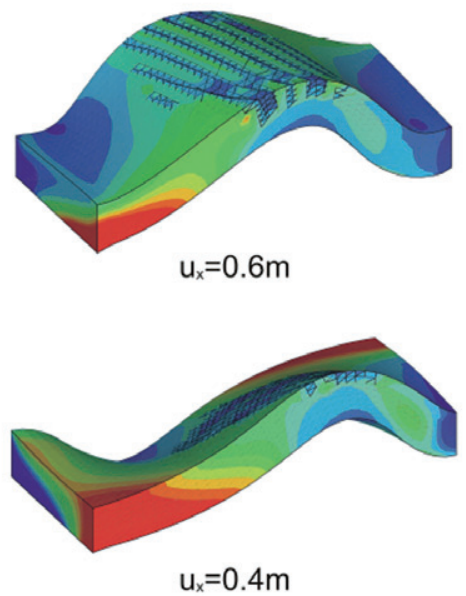

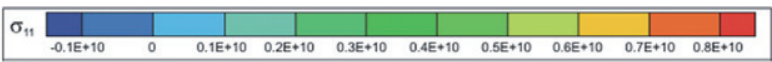

Figure 12. Normal component of Cauchy stress $\sigma_{x x}$ in direction of compression displayed for load cases II-VII at final load stage with seven cracks.

To further quantify the amplification, we analyse the amplification compression relation for all seven load cases I-VII. To this end, we plot the maximum out of plane displacement $u_{z}[\mathrm{~m}]$ at the top centre point of the plate versus the prescribed compressive displacement $u_{x}[\mathrm{~m}]$ in Figure 13, top. For all seven load cases, the amplification $u_{z}$ increases linearly with increasing prescribed compression $u_{x}$ until a critical compression of $u_{x}=0.1 \mathrm{~m}$. Above this critical compression, the amplification-compression behaviour becomes nonlinear and differs significantly for the different load cases. For the sake of comparison, we compute a purely elastic plate without allowing for fracture to quantify the overall influence of failure. Intuitively, one would expect this undamaged plate to display the smallest amplification. Surprisingly, load cases V and VII, however, show a smaller amplification than the elastic reference case. For the load cases V-VII with a shear-to-compression ratio larger than one, we observe a rotation of the plate's crosssection. This rotation is associated with an increase of bending resistance in the outof-plane direction, despite emerging fracture. In addition, load case VII is associated with the transition to a multiple buckling mode which is initiated at an imposed compression of $0.27 \mathrm{~m}$. This folding mode transition associated with the corresponding computational difficulties is the reason why we have limited our analysis to a maximum shearto-compression ratio of $u_{y}: u_{x}=4$. 

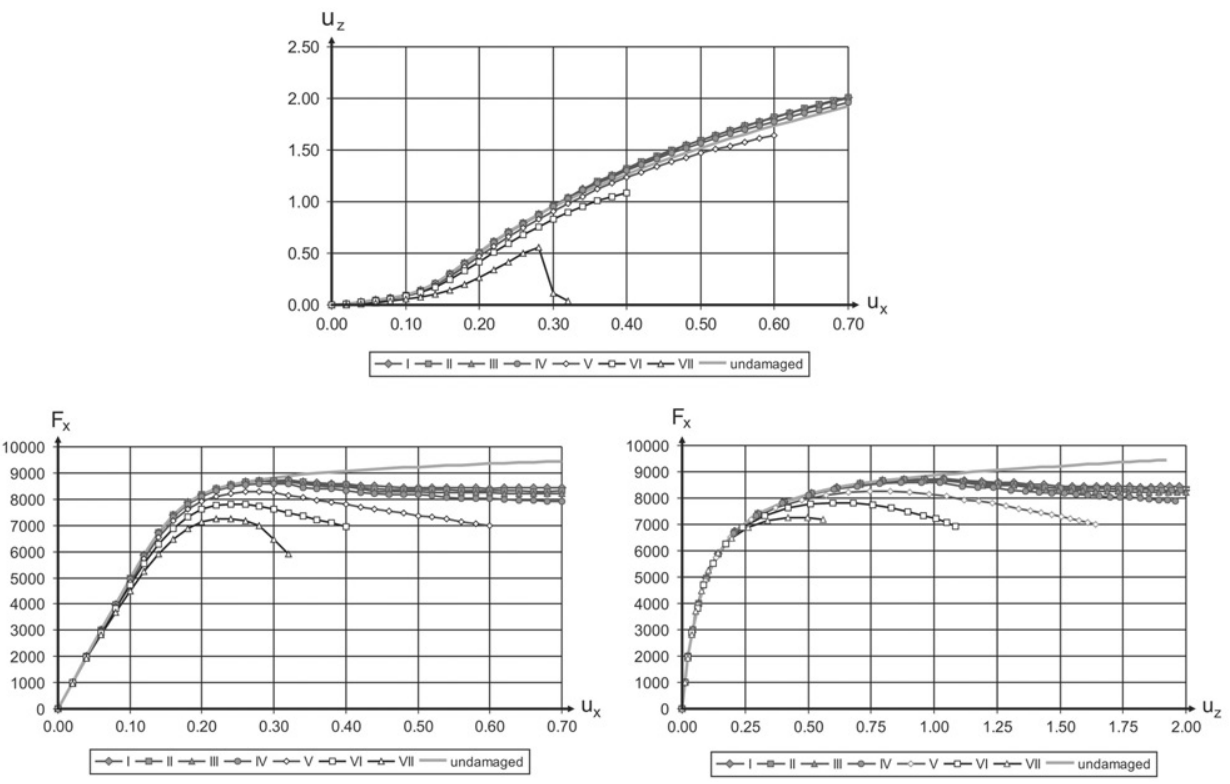

Figure 13. Top: Amplification compression relation $u_{z}[\mathrm{~m}]$ vs. $u_{x}[\mathrm{~m}]$. Bottom left: Compressive force displacement relation $F_{x}[\mathrm{MN}]$ vs. $u_{x}[\mathrm{~m}]$. Bottom right: Compressive force amplification relation Force $F_{x}[\mathrm{MN}]$ vs. $u_{z}[\mathrm{~m}]$.

Figure 13, bottom, gives further insight into the different folding modes associated with the seven load cases. We illustrate the compressive force $F_{x}[\mathrm{MN}]$ versus the compressive displacement $u_{x}[\mathrm{~m}]$ in Figure 13, bottom left, and the compressive force $F_{x}[\mathrm{MN}]$ versus the amplification $u_{z}[\mathrm{~m}]$ in Figure 13, bottom right, respectively. As expected, the undamaged elastic plate of the reference solution shows the highest load carry capacity and its load-displacement relation is monotonically increasing. All plates with emerging fracture show a decreasing load after the limit load is reached. This limit load, however, crucially depends on the shear-to-compression ratio. In accordance with Figure 12, load cases I, II and III with a shear-to-compression ratio below one display an almost identical compression dominated single fold buckling. Accordingly, their forcecompression and force-amplification curves are nearly identical. With an increasing shearto-compression ratio, the overall response becomes more brittle. Remarkably, the limit load under load case VII with a shear-to-compression ratio of $u_{y}: u_{x}=4$ is about $20 \%$ smaller than the limit load for the plain compression case with $u_{y}: u_{x}=0$. Subjected to load case VII, the plate shows a pronounced drop in load-carrying capacity after the limit load is reached. This drastic loss of load carrying capacity associated with the complex multifold failure mechanism could possibly explain the failure of the suggested computational algorithm at even larger shear-compression ratios.

\section{Discussion}

For the first time, we have presented a computational analysis tool that enables the simulation of folding-induced fracturing of geological rock layers in a self-consistent way. 
This tool is based on a novel staggered two-field finite element analysis in terms of the potentially discontinuous deformation field and the scalar-valued crack isosurface. To elaborate the features of the suggested algorithm, we have defined a unique threedimensional model problem of plate folding (or plate buckling). With this model setup, we analyse brittle tensile failure governed by the traditional principle stress based Rankine criterion. In contrast to classical model problems for quasi-brittle materials such as concrete or rock, loading is not applied in the form of tension or bending. Here, we apply a pure displacement driven layer-parallel compression and superpose a gradually varying amount of in-plane shear to systematically study the influence of the stress state on the failure mode. For our particular model problem, brittle failure in the tensile regime of the plate is a mere result of folding or rather buckling, a geometric instability that requires a kinematically exact characterisation of large deformations.

The two most common fracture sets related to folding are both perpendicular to the bedding but either parallel or orthogonal to the fold axes (e.g. [2]). Since both of these fractures open orthogonally to the least principle stress direction, they cannot form simultaneously. In this study, only fractures with an orientation sub-parallel to the fold axes developed. Branching and intersecting cracks are not considered within the present framework. For the present finite element based fracture algorithm, the failure mode is primarily controlled by the principle stress based Rankine failure criterion, the choice of boundary conditions and the magnitude of the failure stress.

In the present study, the elastic plate is free to buckle and not embedded in a mechanically weaker medium. An embedding medium changes the stress magnitude and distribution within the folding layer and may therefore influence the mode of fracturing. However, most probably, the first order results provided by this study will not change dramatically due to an embedding medium.

A component of layer-parallel shearing, or wrenching, results in a systematic variation of the fracture orientation. The results show that above a certain shear-to-compression ratio, shear fractures originate first and not dilational fractures. This shear dominant regime is governed by multiple folding and high crack deviation angles. Below the critical shear-to-compression ratio, however, the crack deviation angle varies linearly with the shear-to-compression ratio. Within this linear regime, the crack deviation angle (i.e. the deviation in orientation of dilational fractures from the fold axis) may be used in the field to estimate the action and magnitude of wrenching during folding. The fracturing during folding changes the effective mechanical properties of the plate, in particular the plate becomes weaker at the outer hinge area and the flexural rigidity becomes smaller at the fold hinge. This weakening would cause an increased amplification for layer-parallel shortening under a constant compressional force.

In this paper, we suggest a nonlinear coupled finite element based algorithm to simulate brittle fracture in folding rocks. An alternative method to study simultaneous folding and fracturing is the discrete element method (e.g. [24]) for which the folding rock is assumed to consist of multiple particles ab-initio. To simulate discrete element based folding and fracturing with an acceptable numerical resolution, however, requires a significantly higher computational effort than using finite elements. Another drawback of discrete element methods is that most established failure criteria based on laboratory rock deformation experiments such as the Rankine criterion are expressed in terms of stress tensor components. These are difficult to define within the discrete element method. Accordingly, the suggested finite element algorithm is believed to provide a stable, robust, 
efficient and powerful analysis tool to study the complex failure phenomena in brittle fracture and folding. The proposed framework can be used to generate individual tables of crack deviation angles versus shear-to-compression ratios for various types of rocks. These tables can potentially be applied in the field to improve the understanding of the evolution and structural development of complex failure patterns in natural rocks. Since these fractures severely influence the permeability of geological structures, this understanding is of crucial importance for industrial applications such as groundwater and hydrocarbon flow.

\section{References}

[1] M.A. Biot, GSA Bulletin 72 (1961) p.1595.

[2] N.J. Price and J.W. Cosgrove, Analysis of Geological Structures, Cambridge University Press, Cambridge, 1990.

[3] S.M. Schmalholz, Y.Y. Podladchikov and J.-P. Burg, J. Geophys. Res. 107 (B1), 2005, doi:10.1029/2001JB000355 (2002).

[4] D.L. Turcotte and G. Schubert, Geodynamcis, John Wiley, New York, 1982.

[5] D.O. Brush and B.O. Almroth, Buckling of Bars, Plates and Shells, McGraw-Hill, New York, 1975.

[6] J.W. Cosgrove and M.S. Ameen, in Forced Folds and Fractures, J.W. Cosgrove and M.S. Ameen eds., Geological Society Special Publication No. 169, London, 2000, p.7.

[7] S. Bergbauer and D.D. Pollard, GSA Bulletin 116 (2004) p.294.

[8] H. Cloos and Z. Deutsch, Geol. Ges. 100 (1948) p.290.

[9] P.L. Hancock, J. Struct. Geol. 7 (1985) p.437.

[10] H. McQuillan, AAPG Bulletin 57 (1973) p.2367.

[11] N.J. Price, Fault and Joint Development in Brittle and Semi-Brittle Rock, Pergamon Press, Oxford, 1966.

[12] D.T. Griggs and J. Handin, in Rock Deformation, D.T. Griggs and J. Handin eds., Geological Society of America Memoir 79, Boulder, 1960, p.347.

[13] T. Belytschko, M. Moës, S. Usui et al., Int. J. Numer. Meth. Eng. 50 (2001) p.993.

[14] J. Dolbow, N. Moës and T. Belytschko, Finite Elements in Analysis and Design 36 (2000) p.235.

[15] G.N. Wells and L.J. Sluys, Int. J. Numer. Meth. Eng. 50 (2001) p.2667.

[16] A. Hansbo and P. Hansbo, Comput. Methods Appl. Mech. Eng. 193 (2004) p.3532.

[17] J. Mergheim, E. Kuhl and P. Steinmann, Int. J. Numer. Meth. Eng. 63 (2005) p.276.

[18] P. Jäger, P. Steinmann and E. Kuhl, Int. J. Numer. Meth. Eng., doi:10.1002/nme.2353.

[19] P. Jäger, P. Steinmann and E. Kuhl, Comput. Mech. 42 (2008) p.395.

[20] J. Mergheim, E. Kuhl and P. Steinmann, Commun. Numer. Meth. Eng. 23 (2007) p.97.

[21] P.M.A. Areias and T. Belytschko, Int. J. Numer. Meth. Eng. 63 (2005) p.760.

[22] T.C. Gasser and G.A. Holzapfel, Comput. Methods Appl. Mech. Eng. 195 (2006) p.5198.

[23] J. Oliver, A.E. Huespe, E. Samaniego et al., Int. J. Numer. Anal. Meth. Geomech. 28 (2004) p.609.

[24] S. Hardy and E. Finch, Basin Res. 17 (2005) p.507. 